\title{
How precision medicine changes medical epistemology: A formative case from Norway
}

\author{
Henrik Vogt ${ }^{1}$ and Bjørn Hofmann ${ }^{2}$ \\ ${ }^{1}$ University of Oslo Faculty of Medicine \\ ${ }^{2}$ Norwegian University of Science and Technology Gjøvik
}

October 6, 2021

\begin{abstract}
Rationale and aims: Precision medicine (PM) raises a key question: How can we know what works when the number of people with a health problem becomes small or one $(n=1)$ ? We here present a formative case from Norway. The Norwegian Board of Health Supervision was faced with a cancer patient, who had improved after treatment with a drug in the private health sector but was refused continued treatment in the public health service due to lack of clinical trial evidence. The Board overturned this decision, arguing that the drug had been unambiguously documented to work in the individual case. We aim to provide an in-depth analysis of this case and The Board's decision and thereby to illustrate and elucidate key epistemological and ethical issues and developments in PM. Method: We provide our analysis and discussion using tools of critical thinking and concepts from philosophy of science and medicine such as uncertainty, evidence, forms of inference and causation. We also examine the case in the light of the history of evidence-based medicine (EBM). Results and discussion: The case reflects an epistemological shift in medicine where PM puts greater emphasis on evidence that arises in individual patients after the treatment is provided over preexisting population-based evidence. PM may rely more heavily on abduction to decide what works and qualitative, rather than quantitative judgments. The case also illustrates a possible shift in the concept of causation from regularity accounts to mechanistic and process accounts. We discuss ethical implications of a shift from more "traditional" to "personalized EBM". Conclusion: A framework that is more based on abductions and evidence arising in the individual case has problems in creating quantifiable, reliable, and generalizable evidence, and in promoting transparency and accountability. PM currently lacks clear criteria for deciding what works in an individual, posing ethical challenges.
\end{abstract}

\section{Hosted file}

20210929 How precision medicine changes medical epistemology- A formative case from Norway Vogt Hofma available at https://authorea.com/users/439869/articles/540679-how-precision-medicinechanges-medical-epistemology-a-formative-case-from-norway 